\title{
Checking for CFFD-Preorder with Tester Processes
}

\author{
Juhana Helovuo and Antti Valmari \\ Tampere University of Technology, Software Systems Laboratory \\ PO Box 553, FIN-33101 Tampere, FINLAND \\ \{juhe, ava\}@cs.tut.fi
}

\begin{abstract}
This paper describes an on-the-fly technique for computing the CFFD-preorder relation on two labelled transition systems (LTSs). CFFD is a process-algebraic semantic model for comparing processes. It is a modification of the CSP model. LTSs are used as a representation of processes. The presented technique is based on transforming the specification process into a special tester process. The tester is then composed in parallel with the processes of the implementation. Violations against the specification are detected as illegal states, deadlocks and livelocks during the computation of the composition. Tester processes are an extension of Brinksma's canonical testers. Using a tester process can be a substantially faster method of computing CFFD-preorder than the previously used method of comparing acceptance graphs.
\end{abstract}

\section{Introduction and Motivation}

This work is based on process-algebraic theories such as Calculus of Communicating Systems (CCS) [11], Communicating Sequential Processes (CSP) [5] [14], and Chaos-Free Failures-Divergences (CFFD) [18,19]. These theories define ways to compare processes. Each of them defines its own notion of equivalence, that is, defines when the behaviours of two processes are considered equivalent.

The semantic model used in this paper is CFFD, which is a further development of CSP. Unlike CSP, the CFFD theory can distinguish between different behaviours after a possible livelock. CSP considers all livelock situations as "chaos" and therefore equivalent, no matter what can happen after the potential livelock. CFFD avoids this problem, hence the name "Chaos-Free Failures-Divergences".

In this paper it is assumed that the processes are formally modelled as labelled transition systems (LTS), or parallel compositions thereof. LTSs resemble nondeterministic automata. The key difference is that an LTS has no accepting states, and, in addition to the normal alphabet, there is a special invisible action $\tau$. $\tau$ resembles the $\varepsilon$ of automata theory, but is usually given more significance.

Many process-algebraic theories contain a preorder relation that can be considered as an implementation relation: a system Sys is a legal implementation of a specification $S p e c$, if and only if Sys $\leq$ Spec. A detailed analysis of many of them is in [9]. The CFFD-preorder " $\leq_{\mathrm{CFFD}}$ " is particularly interesting, because 
- it has nice compositional properties, so it is suitable for compositional verification;

- it handles livelocks in a satisfactory way; and

- it has an interesting connection to ordinary state-based linear-time temporal logic (LTL) [10].

Namely, if Sys $\leq_{\text {CFFD }}$ Spec holds and Spec satisfies an LTL formula, then also Sys satisfies it, provided that the values of the atomic propositions of the formula are fully determined by the non- $\tau$ actions executed so far, and the formula does not contain the "next state" operator [7]. (Notice that Sys may satisfy also formulas that Spec does not satisfy.) This is an important property, because LTL is one of the most important formalisms used for specifying systems, "next state" is usually considered irrelevant in concurrency contexts, and the $\tau$-actions can be chosen according to the atomic propositions of the formula. Furthermore, all major process-algebraic preorders lack this property because of the way they handle livelocks. There are less well known preorders that have the property and some that also preserve deadlocks, but CFFD-preorder can be shown to be the coarsest compositional one among them [7].

CFFD-preorder guarantees that Sys has only "legal" execution traces (those specified by Spec), Sys has only legal refusal sets (a generalization of deadlocks), and Sys can livelock only when specified by Spec. It implies testing preorder, or failure preorder [1].

Checking of Sys $\leq_{\mathrm{CFFD}}$ Spec is PSPACE-complete in the size of Spec. On the other hand, it can be done in low-order polynomial time in the size of Sys. We will develop an algorithm that is exponential in the size of Spec and fast in the size of Sys. It is based on converting Spec into a special tester process, putting it in parallel with $S y s$, and checking for certain conditions when computing the parallel composition of Sys and tester $($ Spec). In addition to being cheap in terms of the size of Sys, the algorithm has the advantage that it can detect errors onthe-fly, that is, during the construction of an LTS for Sys. This is obtained by presenting Sys as a parallel composition of several processes $P_{1}, P_{2}, \ldots, P_{n}$, and computing $P_{1}\left\|P_{2}\right\| \cdots\left\|P_{n}\right\|$ tester $($ Spec $)$ and simultaneously checking for the error detection conditions. As was discussed in detail in [16] Section 4.2, onthe-fly verification may save a lot of effort when analysing erroneous systems, compared to fully computing the LTSs of Spec and Sys before comparing them.

Many of the fundamental ideas presented in this paper are not new. They can be found from the literature on reduction algorithms in classic automata theory, the theory of canonical testers [2], and the CSP model checking in the FDR tool $[13,14]$. However, as far as we know, this is the first time that the theory of tester processes is put together and presented in an organized manner. Furthermore, nontrivial new ideas were needed for adding the handling of divergences in an appropriate way, and, as we pointed out above, divergences are necessary for checking the absence of illegal livelocks.

Other algorithms for checking a preorder that resembles CFFD have been proposed in [3,13]. The [3] approach is exponential also in the size of Sys. The [13] approach is rather similar to ours and has more or less the same complexity. The 
main difference, in addition to the important fact that our approach handles also livelocks, is that some things that the [13] approach postpones to the computation of the parallel composition are done already during the tester construction in our method. Therefore, our method states less requirements to the parallel composition tool. (Please be not confused by the fact that the handling of livelocks causes a rather strict requirement in our method - this should not be taken into account when comparing to [13], because the latter treats livelocks as "chaos".)

The algorithm described in Section 4 has been implemented as an addition to the "Advanced Reachability Analysis" verification tool set, which has been developed by VTT Electronics and the Verification Algorithm Research Group at Tampere University of Technology [17].

Section 2 recalls the basic definitions. In Section 3 the notion of acceptance graphs is introduced. Our new method is described in Section 4.

\section{$2 \quad$ Background}

\subsection{CFFD Semantics}

The CFFD semantics is a semantic model for labelled transition systems (LTS). It is a modification of the CSP model [5] [14]. The CFFD model differs from the CSP model in the handling of (possible) divergences. The CFFD model records the behavior of the system after divergences whereas the CSP model disregards any behavior after the first divergence. The CFFD model is described in detail in [18] and [19]. Here are some definitions of the concepts used in this paper.

Definition 1 (Labelled transition system) LTS $P$ is the 4-tuple $P=(S, \Sigma$, $\Delta, \hat{s})$, where $S$ is the set of states, $\Sigma$ is the finite set of visible action symbols. There is also the invisible action $\tau \notin \Sigma . \Delta \subseteq S \times(\Sigma \cup\{\tau\}) \times S$ is the set of transitions and $\hat{s} \in S$ is the initial state.

In addition to the alphabet symbols in $\Sigma$ there is the invisible action $\tau$. It denotes some execution step which is internal to the system and its execution cannot be directly observed in the execution behavior of the LTS.

Definition 2 (Arrow notation) The following are more convenient notations for executions of an LTS.

$-s-a \rightarrow s^{\prime}$ iff $\left(s, a, s^{\prime}\right) \in \Delta$

$-s-a_{1} a_{2} \ldots a_{n} \rightarrow s^{\prime}$ iff $\exists s_{0}, s_{1}, \ldots, s_{n}: s=s_{0} \wedge s_{0}-a_{1} \rightarrow s_{1}-a_{2} \rightarrow \cdots-a_{n} \rightarrow s_{n} \wedge s^{\prime}=s_{n}$.

- If $\sigma=a_{1} a_{2} \ldots a_{n}$ then $s-a_{1} a_{2} \ldots a_{n} \rightarrow s^{\prime}$ can be written $s-\sigma \rightarrow s^{\prime}$.

$-s-\sigma \rightarrow$ iff $\exists s^{\prime}: s-\sigma \rightarrow s^{\prime}$

$-s=a_{1} a_{2} \ldots a_{n} \Rightarrow s^{\prime}$ iff $s-\tau^{*} a_{1} \tau^{*} a_{2} \tau^{*} \ldots \tau^{*} a_{n} \tau^{*} \rightarrow s^{\prime}$, where each $\tau^{*}$ denotes any sequence of zero or more $\tau$-actions, and none of $a_{i}$ is $\tau$.

$-s=\sigma \Rightarrow$ iff $\exists s^{\prime}: s=\sigma \Rightarrow s^{\prime}$ 
Parallel composition is an operator which combines many LTSs into one. We use a parallel composition operator that synchronizes all visible actions which occur in the alphabets of more than one component LTS.

Definition 3 (Parallel composition) Let $L_{1}=\left(S_{1}, \Sigma_{1}, \Delta_{1}, \hat{s}_{1}\right), \ldots, L_{n}=$ $\left(S_{n}, \Sigma_{n}, \Delta_{n}, \hat{s}_{n}\right)$. Their parallel composition $L_{1}\|\cdots\| L_{n}=(S, \Sigma, \Delta, \hat{s})$, where

$-S=S_{1} \times S_{2} \times \cdots \times S_{n}$

$-\Sigma=\Sigma_{1} \cup \Sigma_{2} \cup \cdots \cup \Sigma_{n}$

- $\left(s, a, s^{\prime}\right) \in \Delta$, where

$s=\left(s_{1}, \ldots, s_{n}\right) \in S \wedge s^{\prime}=\left(s_{1}^{\prime}, \ldots, s_{n}^{\prime}\right) \in S$,

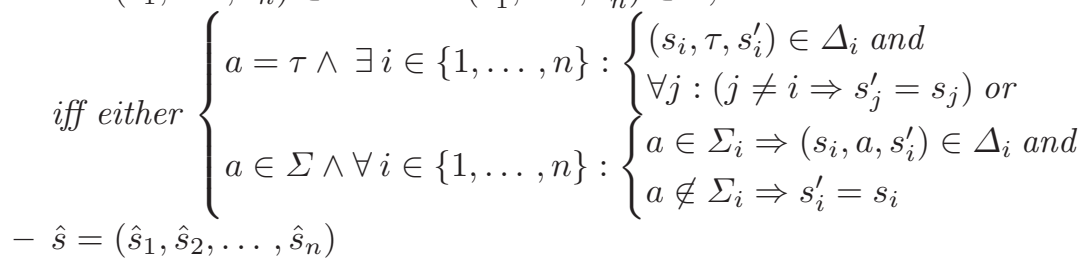

The LTSs generated by this definition of composition contain unreachable states. In practice, these states can be ignored, since they don't affect the behavior of the LTS. In other words, only states $s$ which satisfy $\exists \sigma: \hat{s}-\sigma \rightarrow s$ are important.

Definition 4 (Traces) The set of traces of an $\operatorname{LTS} P=(S, \Sigma, \Delta, \hat{s})$ is $\operatorname{tr}(P)=$ $\left\{\sigma \in \Sigma^{*} \mid \hat{s}=\sigma \Rightarrow\right\}$

Definition 5 (Stable failures) The set of stable failures of an LTS $P$ is the set of pairs sfail $(P)=\left\{(\sigma, R) \in \Sigma^{*} \times 2^{\Sigma} \mid \exists s: \hat{s}=\sigma \Rightarrow s \wedge \forall a \in R \cup\{\tau\}\right.$ : $\neg(s-a \rightarrow)\}$. The set $R$ is called a refusal set.

The number of refusal sets for a given $\sigma$ is often very large. All subsets of a refusal set are also refusal sets, that is $(\sigma, R) \in \operatorname{sfail}(P) \wedge R^{\prime} \subseteq R \Rightarrow\left(\sigma, R^{\prime}\right) \in$ $\operatorname{sfail}(P)$. Therefore, the stable failures with maximal refusal sets with respect to $\subseteq$ completely determine the set of stable failures.

Sometimes it is more convenient to use acceptance sets instead of refusal sets. Acceptance sets are the complements of refusal sets. Complements of the maximal refusal sets are the minimal acceptance sets.

Definition 6 (Deadlock traces) The set of deadlock traces of an LTS $P$ is the set $\operatorname{dltr}(P)=\{\sigma \in \operatorname{tr}(P) \mid \exists s:(\hat{s}=\sigma \Rightarrow s \wedge \forall a \in \Sigma \cup\{\tau\}: \neg(s-a \rightarrow))\}$

Thus

Theorem $1 \operatorname{dltr}(P)=\{\sigma \mid(\sigma, \Sigma) \in \operatorname{sfail}(P)\}$.

Definition 7 (Divergence traces) The set of divergence traces of an LTS P is the set $\operatorname{divtr}(P)=\left\{\sigma \mid \exists s: \hat{s}=\sigma \Rightarrow s \wedge s-\tau^{\omega} \rightarrow\right\}$ 
Here $\tau^{\omega}$ denotes an infinite sequence of invisible events. Similarly, $\Sigma^{\omega}$ denotes all infinite sequences of visible actions.

Traces can be derived from stable failures and divergence traces [15]:

Theorem $2 \operatorname{tr}(P)=\operatorname{divtr}(P) \cup\{\sigma \mid(\sigma, \emptyset) \in \operatorname{sfail}(P)\}$.

Definition 8 (Infinite traces) If $P$ is an LTS, then $\operatorname{inftr}(P)=\left\{\sigma \in \Sigma^{\omega} \mid \hat{s}=\sigma \Rightarrow\right\}$

Definition 9 (Stability) State $s$ is stable iff there are no invisible transitions from it: stable $(s) \Leftrightarrow \neg(s-\tau \rightarrow)$. An LTS P is stable iff its initial state is stable.

Definition 10 (CFFD-equivalence) LTSs $P$ and $Q$ are CFFD-equivalent, written $P \equiv_{C F F D} Q$, iff $\Sigma(P)=\Sigma(Q) \wedge \operatorname{stable}(P)=\operatorname{stable}(Q) \wedge \operatorname{sfail}(P)=$ $\operatorname{sfail}(Q) \wedge \operatorname{divtr}(P)=\operatorname{divtr}(Q) \wedge \operatorname{inftr}(P)=\operatorname{inftr}(Q)$

There are five components $(\Sigma$, stable (), sfail ()$, \operatorname{divtr}()$ and $\operatorname{inftr}())$ in the CFFD-equivalence. In the case of finite state systems the inftr()-component follows from the other components:

Theorem 3 If $P$ is a finite state LTS, then inftr $(P)=\left\{\omega \in \Sigma^{\omega} \mid \forall \sigma<\omega\right.$ : $\sigma \in \operatorname{tr}(P)\}$.

The proof can be found in [19].

The following formulae are intended to illustrate the relation between CFFD and CSP $[12,15]$. Let $\rho \leq \sigma$ denote that $\rho$ is a prefix of $\sigma$. Let $P$ be an LTS. Then its CSP-divergences and CSP-failures are

Definition 11 (CSP-divergences and CSP-failures) $\operatorname{CSPdiv}(P)=\{\sigma \in$ $\left.\Sigma^{*} \mid \exists \rho \in \operatorname{divtr}(P): \rho \leq \sigma\right\}$ and $\operatorname{CSPfail}(P)=\operatorname{sfail}(P) \cup\left(\operatorname{CSPdiv}(P) \times 2^{\Sigma}\right)$.

\subsection{CFFD-Preorder}

The CFFD-preorder is a relation which is used to compare two LTSs. One of the LTSs represents the specification $(S)$ and the other represents the implementation $(I)$. The relation is written $I \leq_{\mathrm{CFFD}} S$.

Definition 12 (CFFD-preorder) If $P$ and $Q$ are LTSs, then $P \leq_{C F F D} Q$ iff 1. $\Sigma(P)=\Sigma(Q)$,

2. $\operatorname{stable}(P) \vee \neg$ stable $(Q),($ or, equivalently, stable $(Q) \Rightarrow \operatorname{stable}(P))$

3. $\operatorname{sfail}(P) \subseteq \operatorname{sfail}(Q)$,

4. $\operatorname{divtr}(P) \subseteq \operatorname{divtr}(Q)$ and

5. $\operatorname{inftr}(P) \subseteq \operatorname{inftr}(Q)$

This relation is both reflexive and transitive, so it is a preorder. The equivalence induced by this preorder is the CFFD-equivalence. That is, $P \leq_{\mathrm{CFFD}}$ $Q \wedge Q \leq_{\mathrm{CFFD}} P \Longleftrightarrow P \equiv_{\mathrm{CFFD}} Q$.

If the LTSs are both finite, then the fifth property follows from the 3rd and 4th. 
Property 1 tells that the implementation and specification should be interested in the same set of actions. Property 2 tells that if the specification is stable, then the implementation must be also stable. In general, the specification may contain more nondeterminism than the implementation but not the other way around. Property 3 states that the implementation is allowed to refuse some set $R \subseteq \Sigma$ of actions after executing a trace $\sigma \in \Sigma^{*}$ only if the specification can execute $\sigma$ and then refuse $R$.

The implementation does not have to refuse everything that the specification may refuse. When considering the acceptance sets instead of refusal sets, the situation is complementary: After executing $\sigma$ the implementation should always be able to accept some superset of one of the specification's acceptance sets. Note that this property only speaks of the stable states.

Property 4 means that the implementation is allowed to diverge (livelock) after executing some trace only if also the specification can diverge after that trace. The implementation may also choose not to livelock even if the specification allows it to do so, but then the implementation refuses at least $\emptyset$ after the trace, which may violate property 3 .

Property 5 is important only in the case of infinite state systems as Theorem 3 states. In [19] this issue is presented in more detail.

Definition 12 (properties 3 and 4) with theorems 1 and 2 implies two additional properties:

Theorem 4 If $P \leq_{C F F D} Q$ then $6 . \operatorname{tr}(P) \subseteq \operatorname{tr}(Q)$ and $\% \cdot \operatorname{dltr}(P) \subseteq \operatorname{dltr}(Q)$

\subsection{The Complexity of Checking CFFD-Preorder}

The checking of equivalence according to a semantics that is based on failures is typically PSPACE-complete [8]. This applies also to CFFD-semantics. Because $P \equiv_{\mathrm{CFFD}} Q \Leftrightarrow P \leq_{\mathrm{CFFD}} Q \wedge Q \leq_{\mathrm{CFFD}} P$, the problem of checking $P \leq_{\mathrm{CFFD}} Q$ must be PSPACE-hard in the size of at least one of $P$ and $Q$. The next theorem states that it is hard in the size of $Q$. On the other hand, as will be described in Section 4.1, it can be done in low-order polynomial time in the size of $P$.

Theorem 5 Checking that $P \leq_{C F F D} Q$ is PSPACE-complete in the size of $Q$.

Proof. If $P \leq_{\mathrm{CFFD}} Q$ does not hold, then at least one of properties 1 to 4 of Definition 12 does not hold. The first two are trivial to check. If the property 3 or 4 does not hold, $P$ and $Q$ have a trace $\sigma$ such that either there is an action $a$ such that $\sigma a \in \operatorname{tr}(P)$ but $\sigma a \notin \operatorname{tr}(Q)$; or there is $A \subseteq \Sigma$ such that $(\sigma, A) \in \operatorname{sfail}(P)$ but $(\sigma, A) \notin \operatorname{sfail}(Q)$; or $\sigma \in \operatorname{divtr}(P)$ but $\sigma \notin \operatorname{divtr}(Q)$. A nondeterministic algorithm can guess the kind of error and then one by one guess the steps of an execution of $P$ that yields $\sigma$ as the trace, and leads $P$ to a state with an illegal next action $a$, refusal set $A$, or divergence. The algorithm simultaneously keeps track of all the states that $Q$ may be in after executing the same trace as $P$ has so far executed.

At some point the algorithm guesses that the execution has been completed. Then it verifies that $P$ can continue with $a$ or refuse $A$ or diverge from its current 
state, but $Q$ cannot from any of the states in its current set of states. Thanks to the interleaving of the steps of $P$ and $Q$, neither the (possibly exponentially long) execution nor its trace $\sigma$ needs to be stored. Keeping track of the set of states of $Q$ requires two or three bits per each state of $Q$. So polynomial space suffices. Thus $P \mathbb{E}_{\mathrm{CFFD}} Q$ can be checked in nondeterministic polynomial space, and NPSPACE is know to be the same as PSPACE.

It remains to be proven that checking $P \leq_{\mathrm{CFFD}} Q$ is PSPACE-hard. The checking whether a nondeterministic finite automaton $N=(S, \Sigma, \Delta, \hat{s}, F)$ accepts all traces (known as "words" in automata theory) in its alphabet is a well-known PSPACE-complete problem. ( $F \subseteq S$ is the set of acceptance states; the other components are like in an LTS; and the automaton accepts a trace if and only if it has an execution that produces that trace and ends in an acceptance state.) This problem can be reduced to the checking of $P \leq_{\mathrm{CFFD}} Q$ in polynomial time as follows.

First, $N$ is converted to the LTS $L_{1}=\left(S_{1}, \Sigma_{1}, \Delta_{1}, \hat{s}\right)$, where $S_{1}=S \cup\left\{s_{\#}\right\}$, $\Sigma_{1}=\Sigma \cup\left\{a_{\#}\right\}, \Delta_{1}=\Delta \cup\left\{\left(s, a_{\#}, s_{\#}\right) \mid s \in F\right\} \cup\left\{\left(s_{\#}, a, s_{\#}\right) \mid a \in \Sigma_{1}\right\}$, and $s_{\#}$ and $a_{\#}$ are new (i.e., $s_{\#} \notin S$ and $a_{\#} \notin \Sigma$ ). That is, a new state is added such that it can be reached from each original acceptance state by $a_{\#}$, and from itself by just any visible action. If $N$ rejects some trace $\sigma$, then $\sigma a_{\#} \notin$ $\operatorname{tr}\left(L_{1}\right)$. Otherwise, $L_{1}$ can execute any $\sigma \in \Sigma^{*}$ in such a way that it can then continue with $a_{\#}$ and any element of $\Sigma_{1}^{*}$. Thus $N$ accepts all traces if and only if $\operatorname{tr}\left(L_{1}\right)=\Sigma_{1}^{*}$.

Next $S_{1}, \Delta_{1}$ and $\hat{s}$ are replaced with $S_{2}=\left\{[[s]] \mid s \in S_{1}\right\}$ and $\Delta_{2}=$ $\left\{\left([[s]], a,\left[\left[s^{\prime}\right]\right]\right) \mid\left(s, a, s^{\prime}\right) \in \Delta_{1}\right\}$ and $\hat{s}_{2}=[[\hat{s}]]$, where $[[s]]=\left\{s^{\prime} \in S_{1} \mid s=\varepsilon \Rightarrow s^{\prime} \wedge\right.$ $\left.s^{\prime}=\varepsilon \Rightarrow s\right\}$, yielding $L_{2}=\left(S_{2}, \Sigma_{1}, \Delta_{2}, \hat{s}_{2}\right)$. In other words, each strongly connected component is collapsed into an individual state, where only $\tau$-transitions are taken into account when checking strong connectivity. Very efficient algorithms are known for this, and $\operatorname{tr}\left(L_{1}\right)$ is not changed, that is, $\operatorname{tr}\left(L_{2}\right)=\operatorname{tr}\left(L_{1}\right)$.

Finally a new initial state and $\tau$-transition are added, a new deadlock state is added and made reachable from any old state by one $\tau$-transition, and all "self-loop" $\tau$-transitions are removed. More formally: $S_{3}=S_{2} \cup\left\{s_{I}, s_{D}\right\}, \Delta_{3}=$ $\Delta_{2} \cup\left\{\left(s_{I}, \tau, \hat{s}_{2}\right)\right\} \cup\left\{\left(s, \tau, s_{D}\right) \mid s \in S_{2}\right\}-\left\{(s, \tau, s) \mid s \in S_{3}\right\}$, and $\hat{s}_{3}=s_{I}$, where $s_{I} \notin S_{2}$ and $s_{D} \notin S_{2}$. This conversion leaves $\operatorname{tr}\left(L_{2}\right)$ intact, and ensures that stable $\left(L_{3}\right)=$ false, $\operatorname{sfail}\left(L_{3}\right)=\operatorname{tr}\left(L_{3}\right) \times 2^{\Sigma_{1}}$, and $\operatorname{divtr}\left(L_{3}\right)=\emptyset$.

Consider the LTS $L^{\prime}=\left(\left\{s_{1}, s_{2}\right\}, \Sigma_{1}, \Delta^{\prime}, s_{1}\right)$, where $\Delta^{\prime}=\left\{\left(s_{1}, \tau, s_{2}\right)\right\} \cup$ $\left\{\left(s_{1}, a, s_{1}\right) \mid a \in \Sigma_{1}\right\}$. We have stable $\left(L^{\prime}\right)=$ false, sfail $\left(L^{\prime}\right)=\Sigma_{1}^{*} \times 2^{\Sigma_{1}}$, and $\operatorname{divtr}\left(L^{\prime}\right)=\emptyset$. Therefore, $L^{\prime} \leq_{\mathrm{CFFD}} L_{3}$ holds if and only if $\Sigma_{1}^{*} \subseteq \operatorname{tr}\left(L_{3}\right)$. On the other hand, $\operatorname{tr}\left(L_{3}\right) \subseteq \Sigma_{1}^{*}$ by definition, and $\operatorname{tr}\left(L_{3}\right)=\operatorname{tr}\left(L_{1}\right)$ by construction. So $L^{\prime} \leq_{\mathrm{CFFD}} L_{3}$ holds if and only if $\operatorname{tr}\left(L_{1}\right)=\Sigma_{1}^{*}$, which, in turn, was shown equivalent to $N$ accepting all traces in its alphabet. 


\section{Acceptance Graphs}

In order to check whether the relation $P \leq_{\mathrm{CFFD}} Q$ holds, we have to check if the properties 1-5 of Definition 12 hold for them. If we assume that $Q$ does not have an infinite number of states, then property 5 need not be checked.

Properties 1 and 2 are simple. Equivalence of alphabets is just a set comparison. The values of stable $(P)$ and stable $(Q)$ can be obtained by inspecting the output transitions of the initial states of $P$ and $Q$, and from then on the check of property 2 consists of simple Boolean arithmetic.

To check for properties 3 and 4 we rely on the idea of acceptance graphs, which are a modification of acceptance trees of [4]. They are deterministic LTSs with some additional information.

Definition 13 (Acceptance Graph) An acceptance graph (AG) is the 7tuple $(S, \Sigma, \delta, \hat{s}$, accsets, divok, stable $)$, where

$-S$ is the set of states.

$-\Sigma$ is the alphabet.

$-\delta$ is a set of transitions, unlike in LTSs, it is a partial function $S \times \Sigma \rightarrow S$.

$-\hat{s} \in S$ is the initial state.

- accsets $(s)$ is an attribute of each state in $S$. Its value is a set of subsets of $\Sigma$ such that no set is a subset of another. That is, if accsets $(s)=$ $\left\{A_{1}, A_{2}, \ldots, A_{k}\right\}$, then $A_{i} \nsubseteq A_{j}$ whenever $i \neq j$.

- divok $(s)$ is a Boolean attribute of each state in $S$.

- stable is a Boolean attribute of the entire $A G$.

Because an acceptance graph is deterministic, it can be exponentially bigger than a CFFD-equivalent LTS. Acceptance graphs may thus be expensive to use.

Acceptance graphs and their construction are described in more detail in [18]. Now we will only briefly review the structure of an AG.

An acceptance graph can represent the same information as the CFFD-model of finite LTSs, namely the alphabet, stability, stable failures, and divergence traces. The main difference is that an AG contains no invisible transitions and it is deterministic.

Since the AG is deterministic, each trace $\sigma$ leads to a unique state $s$. If $\sigma \in \operatorname{divtr}(P)$ in the LTS representation, then the corresponding state in the AG has its divok $=$ true. The stable failures are stored as minimal acceptance sets in each state. If there are stable failures $\left(\sigma, R_{1}\right),\left(\sigma, R_{2}\right), \ldots$ associated with trace $\sigma$, then we take the maximal ones among the refusal sets $R_{1}, R_{2}, \ldots$, compute their complements in order to save space and store these complements (minimal acceptance sets) as an attribute to the AG state $s$ reachable with trace $\sigma$. The initial stability information is stored as a separate attribute of the entire AG.

\section{Checking CFFD-Preorder with Tester Processes}

\subsection{Use of the Tester}

A tester process is an augmented LTS which can be used to check CFFD-preorder $P \leq_{\mathrm{CFFD}} Q$ without determinising $P$. Like in the previous checking method, the 
parts 1 and 2 of Definition 12 are checked separately. The parts 3 and 4 are checked by computing the parallel composition of the tester and the original LTS $P$. Any violations of the conditions 3 and 4 for CFFD-preorder can be seen as a result of this construction.

The tester is "almost" deterministic. Given any trace $\sigma$, either the tester cannot execute it at all, or the tester is in a unique state immediately after executing $\sigma$. From this state there may be zero or more $\tau$-transitions, but their end states are stable.

In addition to the components of a normal LTS, the tester will have up to three checks in each of its states. These may be considered as Boolean values attached to the states. Each state can be marked as a rejection state, deadlock rejection state or livelock rejection state, or any combination of these. The values of these flags on the state $s$ are given by the functions reject $(s), \operatorname{DL}-\operatorname{reject}(s)$ and LL-reject $(s)$. They should be observed when constructing the parallel composition of the tester and the implementation under test.

If the parallel composition ever reaches a state where the tester is in a rejection state, it is considered an error of the implementation. Similarly, if the parallel composition deadlocks when the tester is in a deadlock rejection state, or livelocks when the tester is in a livelock rejection state, then these are considered illegal behavior of the implementation.

Violations against rejection and deadlock rejection states are easy to detect on-the-fly, that is, during the construction of the parallel composition. An efficient algorithm for on-the-fly detection of also violations against livelock rejection states was described in [20]. The algorithm is based on constructing the parallel composition in a certain order, and monitoring for loops consisting solely of $\tau$-transitions. Also the "non-progress cycle" detection algorithm of [6] can be used for the task, but [16] argues that it is usually less efficient.

\subsection{Construction of the Tester}

We shall present an algorithm that converts the acceptance graph of $Q$ into a tester process tester $(Q)$. It needs the notion of the minimal mirror collection [2] of a collection of sets.

Definition 14 (Set mirroring) Let $\mathbb{A}=\left\{A_{1}, A_{2}, \ldots, A_{n}\right\}$, where $A_{i} \subseteq \Sigma$ when $i=1, \ldots, n$ be a collection of subsets of $\Sigma$. Define intersect $(\mathbb{A})=\{B \subseteq$ $\left.\Sigma \mid \forall j \in\{1, \ldots, n\}: A_{j} \cap B \neq \emptyset\right\}$. The minimal mirror collection of $\mathbb{A}$ is the set of the minimal members of intersect $(\mathbb{A})$ :

$\operatorname{Mirror}(\mathbb{A})=\left\{M \in \operatorname{intersect}(\mathbb{A}) \mid \neg \exists M^{\prime}: M^{\prime} \subset M \wedge M^{\prime} \in \operatorname{intersect}(\mathbb{A})\right\}$.

Notice that this definition implies $\operatorname{Mirror}\left(\left\{a_{1}, a_{2}, \ldots, a_{n}\right\}\right)=\left\{\left\{a_{1}\right\},\left\{a_{2}\right\}\right.$, $\left.\ldots,\left\{a_{n}\right\}\right\}, \operatorname{Mirror}(\emptyset)=\{\emptyset\}$ and $\operatorname{Mirror}(\{\emptyset\})=\emptyset$. The name suggests that the mirror image of a mirror image of a collection should be the original collection. This is not true in general, but holds if no set in the original collection is a subset of another. 


\section{Algorithm 1 Tester LTS Construction}

Input: An acceptance graph $A G(Q)=\left(S_{Q}, \Sigma_{Q}, \Delta_{Q}, \hat{s}_{Q}\right.$, accsets $_{Q}$, divok $_{Q}$, stable $\left._{Q}\right)$ of LTS $Q$.

Create a new state $s_{\text {fail }}$; $S_{Q}^{\prime}:=S_{Q} \cup\left\{s_{\text {fail }}\right\}$

$\operatorname{reject}\left(s_{\text {fail }}\right):=\operatorname{true} ; \operatorname{LL}-$ reject $\left(s_{\text {fail }}\right):=\operatorname{true} ; \operatorname{DL}-\operatorname{reject}\left(s_{\text {fail }}\right):=\operatorname{true}$

For each state $s \in S_{Q}$ such that $s \neq s_{\text {fail }}$ :

$\operatorname{reject}(s):=$ false $;$ LL-reject $(s):=\neg \operatorname{divok}(s)$

DL-reject $(\mathrm{s}):=\left(\operatorname{accsets}_{Q}(s) \neq\{\emptyset\}\right)$

For each $a \in \Sigma_{Q}$ such that $\neg(s-a \rightarrow)$ :

Create a new transition $s-a \rightarrow s_{\text {fail }}$.

end

$M:=\operatorname{Mirror}(\operatorname{accsets}(s))$

For each $A \in M$ :

Create a new state $s_{A}$.; $S_{Q}^{\prime}:=S_{Q}^{\prime} \cup\left\{s_{A}\right\}$

Create a new transition $s-\tau \rightarrow s_{A}$.

$\operatorname{reject}\left(s_{A}\right):=$ false $; \operatorname{DL}-\operatorname{reject}\left(s_{A}\right):=\operatorname{DL}-\operatorname{reject}(s)$

LL-reject $\left(s_{A}\right):=\operatorname{LL}-\operatorname{reject}(s)$

For each $a \in A$ and $s^{\prime} \in S_{Q}$ such that $s-a \rightarrow s^{\prime}$ :

Create a new transition $s_{A}-a \rightarrow s^{\prime}$.

Mark the transition $s-a \rightarrow s^{\prime}$ for removal.

end

end

Remove marked transitions.

end

Output: tester $(Q)=\left(S_{Q}^{\prime}, \Sigma_{Q}, \Delta_{Q}, \hat{s}_{Q}\right.$, reject, DL-reject, LL-reject $)$

end

\section{Theorem 6}

1. If $A_{1} \subseteq A$, then $\operatorname{Mirror}\left(\left\{A_{1}, A_{2}, \ldots, A_{k}, A\right\}\right)=\operatorname{Mirror}\left(\left\{A_{1}, A_{2}, \ldots, A_{k}\right\}\right)$.

2. If no member of $\mathbb{A}$ is a subset of another, then Mirror $(\operatorname{Mirror}(\mathbb{A}))=\mathbb{A}$.

The proof is skipped because of lack of space.

We are now ready to present the tester construction algorithm. It is the enclosed Algorithm 1. It works by adding new states and transitions to $A G(Q)$, deleting some old transitions, and setting the reject- etc. flags of all states. These operations are guided by the accsets- and divok-attributes of $A G(Q)$.

In theory, the reject flag is not necessary. The only state where it is on, namely $s_{\text {fail }}$, has no output transitions. Furthermore, the tester has the same visible actions as the specification $Q$ and the implementation $P$. Therefore, if $P \|$ tester $(Q)$ reaches a state where tester $(Q)$ is in $s_{\text {fail }}$, then it can continue only with invisible actions. If it can do an infinite number of them, then it livelocks, otherwise it eventually deadlocks. Thus the reaching of $s_{\text {fail }}$ would be detected as a violation against LL-reject $\left(s_{\text {fail }}\right)$ or DL-reject $\left(s_{\text {fail }}\right)$. However, the reject flag is extremely easy to implement, and it makes it possible to catch the error sooner. Early catching of errors is a virtue of on-the-fly verification. 


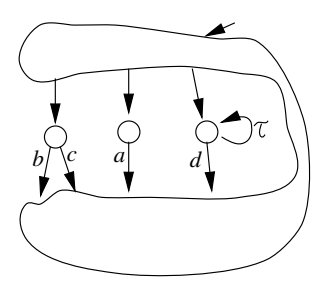

(a) Specification LTS Spec

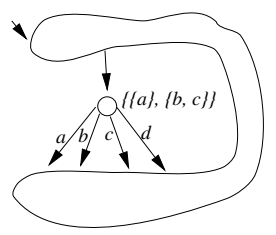

(b) Acceptance Graph $A G($ Spec $)$

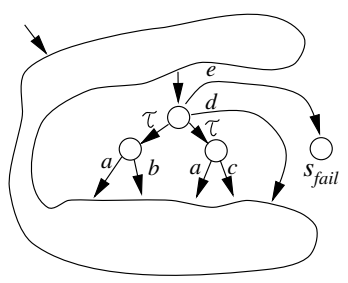

(c) Tester

LTS tester (Spec)

Fig. 1. Stages of the tester construction

Also the DL-reject-flags are unnecessary in theory, but helpful in practice. They could be avoided by adding the transition $s-\tau \rightarrow s$ to every original state $s$ of $A G(Q)$ when starting to process $s$ in the algorithm, and treating every state as a DL-reject-state. Then the parallel composition cannot deadlock in $s$ because the tester can livelock there; it can deadlock only when the tester is in some state $s_{A}$ that the algorithm added to cater for a set $A$ in $\operatorname{Mirror}(\operatorname{accsets}(s))$. If the specification allows deadlocking, then $\operatorname{accsets}(s)=\{\emptyset\}$ and Mirror $(\operatorname{accsets}(s))=\emptyset$, so there will be no $s_{A}$. However, letting the tester livelock would complicate the use of the LL-reject-flags, as one would then have to distinguish those livelocks that are not due to the tester. It would also cause a small increase in the size of the parallel composition LTS.

The construction of the tester may be sped up by minimizing $A G(Q)$ [18] before tester construction.

\subsection{An Example Tester}

The LTS Spec in Figure 1 contains three explicitly shown states, which represent possible states after some trace $\sigma$. All three are reachable with the same trace from the initial state somewhere in the "cloud". The alphabet of this specification is $\Sigma=\{a, b, c, d, e\}$.

In the acceptance graph fragment all three states are combined into one, since they are reachable by the same trace and different stable failures are represented by different acceptance sets in this state. Notice that the state with divergence is unstable and therefore does not generate an acceptance set of its own. However, the divergence state causes the divok-flag of the AG state to be set. The mirror image of these acceptance sets is $\operatorname{Mirror}(\{\{a\},\{b, c\}\})=\{\{a, b\},\{a, c\}\}$.

The tester generated from the acceptance graph shows a new "child" state and a $\tau$-transition to it for each mirrored acceptance set. The visible transitions from the original state have been moved and possibly duplicated to begin from the child states. The transition labelled $d$ is not a member of any acceptance set, 
so it has been left intact. The trace $\sigma e$ is illegal, so that trace leads the tester immediately to the failure state $s_{\text {fail }}$.

\subsection{How Does the Tester Work}

Consider the parallel composition of a tester with another LTS. From it one should be able to tell if any of the properties of the CFFD-preorder are violated. In fact, $\operatorname{sfail}(P) \subseteq \operatorname{sfail}(Q)$ and $\operatorname{divtr}(P) \subseteq \operatorname{divtr}(Q)$ are the only interesting properties, since the alphabet and stability are easy to check without testers, and infinite traces are relevant only if $Q$ has infinitely many states.

Traces First of all, the parallel composition can verify $\operatorname{tr}(P) \subseteq \operatorname{tr}(Q)$. Due to its construction, $A G(Q)$ has the same set of traces as $Q$. If $P$ has a finite trace $\sigma_{\text {fail }}$ that $Q$ cannot execute, then it has a (possibly empty) longest prefix $\sigma_{\text {fail }}^{\prime} \leq \sigma_{\text {fail }}$ which is a trace of both processes. After executing $\sigma_{\text {fail }}^{\prime}, A G(Q)$ will be in a state where it cannot continue with the next visible action in $\sigma_{\text {fail }}$. An attempt to execute this action will lead tester $(Q)$ immediately to $s_{\text {fail }}$. On the other hand, because $A G(Q)$ is deterministic and tester $(Q)$ inherits its structure from $A G(Q), s_{\text {fail }}$ can be reached only by an illegal trace. Therefore, $\operatorname{tr}(P) \nsubseteq \operatorname{tr}(Q)$ if and only if the parallel composition will contain a transition to $s_{\text {fail }}$.

Divergence Traces Since $A G(Q)$ is deterministic, each of its traces uniquely determines a state, where execution ends up after that trace. The LTS $Q$ can diverge after some trace $\sigma$ iff $A G(Q)$ has its divok-flag set in the state $s$ determined by $\sigma$. The tester constructed using the above algorithm has all the states of $A G(Q)$ and some more. In the tester, the trace $\sigma$ leads to state $s$ or one of its "child states" $s_{A}$ added during the algorithm. In all these states the flag LL-reject has the opposite value from $\operatorname{divok}(s)$ in $A G(Q)$.

This means that if the testee process $P$ in the composition has a divergence after $\sigma$, and if $\sigma$ is not rejected as an illegal trace, then the parallel composition will livelock after $\sigma$. If $\sigma$ is in $\operatorname{divtr}(Q)$ (legal divergence), then the LL-reject-flags in the states immediately after $\sigma$ are off, so no error is declared. In the opposite case, the tester component is in a livelock rejection state, so an illegal livelock will be detected during the computation of the parallel composition.

Stable Failures We still have the check $\operatorname{sfail}(P) \subseteq \operatorname{sfail}(Q)$. If $(\sigma, R) \in \operatorname{sfail}(P)$ but $\sigma \notin \operatorname{tr}(Q)$, then $\sigma$ is an illegal trace and will be detected as such. Therefore, let us from now on assume that $\sigma \in \operatorname{tr}(Q)$ but $(\sigma, R) \notin \operatorname{sfail}(Q)$.

Let $s_{P}$ be a stable state of $P$ that can be reached with $\sigma$ and refuses all actions in $R$. Let $R_{1}, \ldots, R_{n}$ be the maximal refusal sets of $Q$ after $\sigma$. Because the refusal of $R$ is illegal, for each $i, R$ contains an action $a_{i}$ that is not present in $R_{i}$. Thus $a_{i} \in \Sigma-R_{i}$, and we can reason that each minimal acceptance set of $A G(Q)$ after $\sigma$ contains an action $a_{i}$ that is also in $R$. The mirror image of the collection of the minimal acceptance sets contains a subset $A$ of the set of those actions, so that $A \subseteq\left\{a_{1}, \ldots, a_{n}\right\}$. The tester has a state $s_{A}$ that is reachable with $\sigma$ and offers precisely the actions in $A$ as the next actions. Because $s_{P}$ 
refuses all of $a_{1}, \ldots, a_{n}$ and both $s_{P}$ and $s_{A}$ are stable, the parallel composition is in a deadlock. Because refusing of $R$ is illegal, we see that $\Sigma$ is not a refusal set of $Q$ after $\sigma$, so the collection of minimal acceptance sets is not $\{\emptyset\}$, its mirror image is not $\emptyset$, and DL-reject $\left(s_{A}\right)=$ true. Thus the deadlock is illegal, and the error is detected. We have now shown that all illegal stable failures are detected.

It remains to be proven that if the parallel composition deadlocks while the tester is in a deadlock rejection state, then $P$ has an illegal stable failure. The deadlock state is of the form $\left(s_{P}, s_{Q}\right)$, and is reached by some common trace $\sigma$ of $P$ and $Q$. The state $s_{Q}$ is either $s_{\text {fail }}$, or one of the $s_{A}$-states added by the tester construction algorithm, or one of the original states of $A G(Q)$. If it is $s_{\text {fail }}$, then already $\sigma$ is illegal. An original state can be a component of a deadlock state only if it has no $\tau$-transitions to the $s_{A}$-states. This is possible only if $\operatorname{Mirror}\left(\operatorname{accsets}\left(s_{Q}\right)\right)=\emptyset$, in which case $\operatorname{accsets}\left(s_{Q}\right)=\{\emptyset\}$, so $\operatorname{DL}-\operatorname{reject}\left(s_{Q}\right)=$ false, and no error is reported. Therefore, assume from now on that $s_{Q}$ is one of the $s_{A}$-states. It corresponds to some set $A$ in $\operatorname{Mirror}\left(\operatorname{accsets}\left(s_{Q}\right)\right)$. By construction, $A$ contains at least one action from each minimal acceptance set of $Q$ after $\sigma$. Thus for each refusal set $R_{i}, 1 \leq i \leq n$, of $Q$ after $\sigma$ there is $a_{i} \in A$ such that $a_{i} \notin R_{i}$. However, because $\left(s_{P}, s_{Q}\right)$ is a deadlock, $s_{P}$ refuses all of $a_{i}$. Thus $s_{P}$ refuses $\left\{a_{1}, \ldots, a_{n}\right\}$ that is not a refusal set of $s_{Q}$.

\subsection{Implementation Considerations}

The tester construction algorithm is otherwise quite straightforward to implement, but it contains operations on sets of subsets of $\Sigma$. Choosing appropriate data structures is important for fast set operations. The most effective set representation in a typical computer depends on the size of $\Sigma$. For a small $\Sigma$ a bit vector representation is most efficient.

Excluding the handling of the acceptance sets (the "for each $A \in M$ " loop and the preceding line, which does set mirroring), the complexity of the algorithm is of the order $|\Sigma||S|$. The cost of processing the acceptance sets, and the number of the states and transitions in the constructed tester, depend greatly on the results of the acceptance set mirroring. If the average number of sets in the mirror images is high, then the resulting tester will be large.

Suppose $\Sigma=\left\{a_{1}, a_{2}, \ldots, a_{2 n}\right\}$, where $a_{i} \neq a_{j}$ whenever $i \neq j$. Then some state may have the acceptance sets $\mathbb{A}=\left\{\left\{a_{1}, a_{2}\right\},\left\{a_{3}, a_{4}\right\}, \ldots,\left\{a_{2 n-1}, a_{2 n}\right\}\right\}$. Because all the elements in these $n$ sets are different, the mirror image will be $\left\{a_{1}, a_{2}\right\} \times\left\{a_{3}, a_{4}\right\} \times \cdots \times\left\{a_{2 n-1}, a_{2 n}\right\}$. The number of these sets is $2^{n}$ or $2^{\left(\frac{|\Sigma|}{2}\right)}$. Therefore, any algorithm for computing the mirror image has a worst-case exponential run time with respect to the size of $\Sigma$.

A brute-force method for computing $\operatorname{Mirror}\left(\left\{A_{1}, A_{2}, \ldots, A_{n}\right\}\right)$ is to first compute the Cartesian product $A_{1} \times A_{2} \times \cdots \times A_{n}$. The result is formally a set of vectors, but these vectors can be converted into sets by dropping the duplicate elements and disregarding the order of elements. Then we only have to find the minimal ones among these sets. This can be done simply by comparing each set to all the others and removing the set if any of its proper subsets is found. 
The computation of a long chain of Cartesian products can be done one product at a time. In this case the computation can be optimized by checking for minimality already at this stage, and discarding sets which have subsets in the already-computed result.

\subsection{Measurements}

The tester construction algorithm has been implemented as an extension to the "Advanced Reachability Analysis" verification tool set [17]. The following measurements are intended to give only a rough estimate of the time consumed by the tester construction algorithm starting from the acceptance graph. The time indicated includes inputting the acceptance graph from a file, constructing the tester and outputting the result into a file. The measurements were made on a Sun SPARCStation 5 with a $70 \mathrm{MHz}$ sparc CPU. The time program provided with the operating system (Solaris 2.5) was used to measure program run times. The first test case is a simple artificial example LTS, the second case is a model of the alternating bit protocol with buffers and the third test case is an incorrect version of the same protocol.

\begin{tabular}{|c|c|c|c|c|}
\hline \multicolumn{2}{|c|}{ Test case } & Simple & $\mathrm{ABP}$ & $\mathrm{BABP}$ \\
\hline \multicolumn{2}{|c|}{ Size of $\Sigma$} & 5 & 5 & 13 \\
\hline \multirow[t]{2}{*}{ Specification AG } & Size of $S$ & 5 & 150 & 1500 \\
\hline & Size of $\Delta$ & 7 & 270 & 3600 \\
\hline \multirow[t]{2}{*}{ Tester LTS } & Size of $S$ & 10 & 310 & 4100 \\
\hline & Size of $\Delta$ & 30 & 750 & 21000 \\
\hline \multicolumn{2}{|c|}{ CPU time used } & $0.2 \mathrm{~s}$ & $1.7 \mathrm{~s}$ & $31 \mathrm{~s}$ \\
\hline \multicolumn{2}{|c|}{ Real time used } & $0.4 \mathrm{~s}$ & $1.9 \mathrm{~s}$ & $32 \mathrm{~s}$ \\
\hline
\end{tabular}

\section{Conclusions}

We have developed an on-the-fly CFFD-preorder verification method which is based on tester processes. Tester processes are ordinary LTSs extended with three kinds of error detection states: reject, reject if deadlock, and reject if livelock. The implementation process is represented as an LTS or a parallel composition of several LTSs. The specification LTS is converted into a tester by determinising, mirroring of the refusal information, adding new states and $\tau$-transitions, and declaring certain states as error detection states. Implementation errors according to $\leq_{\mathrm{CFFD}}$ can be detected on-the-fly with an ordinary LTS parallel composition algorithm augmented with three relatively easy checks based on the local state of the tester. The check for livelocks uses an algorithm that has been published earlier, [6] or [20].

The use of testers requires computationally expensive things to be done only to the specification LTS. A software tool for computing these tester processes from specification LTSs has been implemented. 


\section{Acknowledgments}

This research was funded by the National Technology Agency of Finland (Tekes) in conjunction with the RATE-project, which studies automated testing of reactive systems; and by the Academy of Finland, project "Software Verification with CFFD-Semantics".

\section{References}

1. E. Brinksma, G. Scollo, and C. Steenbergen. LOTOS specifications, their implementations and their tests. Protocol Specification, Testing and Verification VI, IFIP 1987, pages 349-360, 1987. 284

2. Ed Brinksma. A theory for the derivation of tests. In Protocol Specification, Testing and Verification VIII, pages 63-74. North-Holland, 1989. 284, 291

3. Rance Cleaveland and Matthew Hennessy. Testing equivalence as a bisimulation equivalence. Formal Aspects of Computing, 5(1):1-20, 1993. 284

4. M. Hennessy. Acceptance trees. Journal of the ACM 32(4), pages 896-928, 1985. 290

5. C.A.R. Hoare. Communicating Sequential Processes. Prentice-Hall, 1985.283 , 285

6. Gerard J. Holzmann. Design and Validation of Computer Protocols. Prentice Hall, 1990. 291, 296

7. Roope Kaivola and Antti Valmari. The weakest compositional semantic equivalence preserving nexttime-less linear temporal logic. In CONCUR '92: Third International Conference on Concurrency Theory, volume 630 of Lecture Notes in Computer Science, pages 207-221. Springer-Verlag. 284

8. Paris C. Kanellakis and Scott A. Smolka. CCS expressions, finite state processes, and three problems of equivalence. Information and Computation, 86(1):43-68, May 1990. 288

9. Guy Leduc. On the Role of Implementation Relations in the Design of Distrubuted Systems using LOTOS. PhD thesis, Université de Liège, July 1990. 283

10. Z. Manna and A. Pnueli. The Temporal Logic of Reactive and Concurrent SystemsSpecification. Springer-Verlag, 1992. 284

11. R. Milner. Communication and Concurrency. Prentice-Hall, 1989. 283

12. Ernst-Rüdiger Olderog. TCSP: Theory of communicating sequential processes. In Petri Nets: Applications an Relationships to Other Models of Concurrency, volume 255 of Lecture Notes in Computer Science, pages 441-465. Springer-Verlag, July 1986. 287

13. A. W. Roscoe. Model-checking CSP. In A Classical Mind: Essays in Honour of C. A. R. Hoare, pages 353-378. Prentice-Hall, 1994. 284, 285

14. A. W. Roscoe. The Theory and Practice of Concurrency. Prentice-Hall, 1998. 283, 284,285

15. A. Valmari. Compositionality in state space verification methods. In Application and Theory of Petri Nets 1996, 17th International Conference, volume 1091, pages 29-56, 1996. 287

16. A. Valmari. The state explosion problem. In Lectures on Petri Nets I: Basic Models, volume 1491, pages 429-528, 1998. 284, 291 
17. Antti Valmari, Jukka Kemppainen, Matthew Clegg, and Mikko Levanto. Putting advanced reachability analysis techniques together: the "ARA" tool. In FME'93: Industrial-Strength Formal Methods, pages 597-616. Formal Methods Europe, Springer-Verlag, April 1993. Lecture Notes in Computer Science 670. 285, 296

18. Antti Valmari and Martti Tienari. An improved failures equivalence for finitestate systems with a reduction algorithm. In Proceedings of the IFIP WG 6.1 Eleventh International Symposium on Protocol Specification, Testing and Verification, Stockholm, Sweden, pages 3-18. North-Holland, 1991. 283, 285, 290, 293

19. Antti Valmari and Martti Tienari. Compositional failure-based semantic models for Basic LOTOS. Formal Aspects of Computing, 7:440-468, 1995. 283, 285, 287, 288

20. Valmari, A. On-the-fly verification with stubborn sets. In Proc. of the 1993 Workshop on Computer-Aided Verification, 1993. 291, 296 